CONTEXTUAL GRAMMARS

- Solomon Marcus -

INTERNATIONAL CONFERENCE

ON

C"OMPUTATIONAL LIN"N"UISTICS
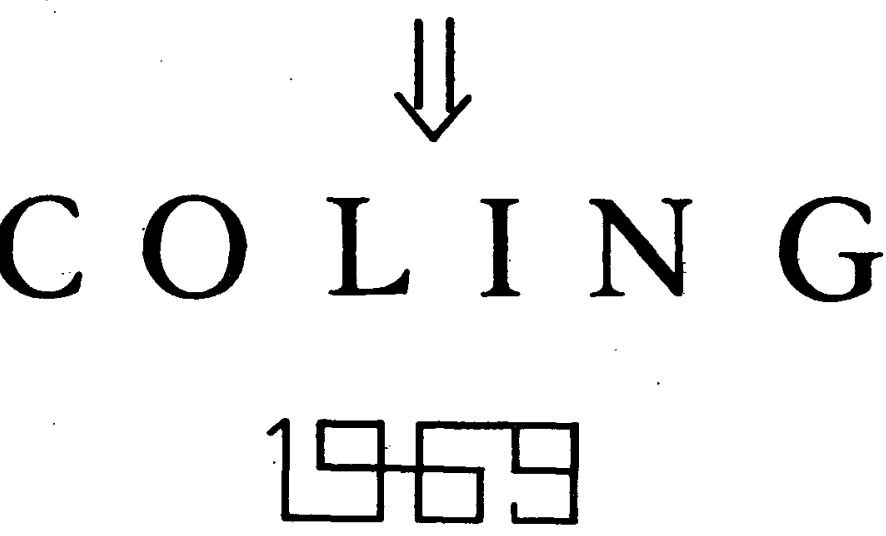

RESBARCH GROUP FOR QUANTITATIVE LINGUISTICS

Addreas: Fack Stockholm 10, SmEDEN 


\section{CONTEXTUAL GRAMMARS}

- Solomon Marcus -

Institutul de Matematica Str, Mihai Eminescu, 47 Bucharest 9, ROMANIA

In the following, we shall introduce a type of generative grammars, called contextual grammars. They are not comparable With regular grámiars. But every language generated by a contextual gramar is a context-eree language. Generalized contextual gramuars are introduced, which may generate non-context-free languages.

Let $\mathrm{V}$ be a finite non-void set ; $\mathrm{Y}$. is called yocabulary. Hery fimite sequence of elements in $y$ is said to be a string on v. Given a string $x={ }_{1} a_{2} \ldots a_{n}$, the number $n$ is called the length of $x$. The string of length zero is called the nulstring and is denoted by $\omega$. Any set of strings on $v$ is called a language on $V$. The set of all strings on $V$ (the nullstring inclusively) is called the universal Janguage on v. By $\mathbf{a}^{\mathbf{n}}$ - we denoite the string $\mathbf{a} . . . a$, where $a$ is iterated $\mathbf{n}$ times.

Any ordered pair 〈u, v> of strings on $V$ is said to be a context on V. The. - string $x$ is admitted by the context $\langle u, v\rangle$ with respect to the language I if $11 \times v \in I$.

Let $I_{1}$ be a finite set of strings on the vocabulary $y$. and let $($ be a finite set of contexts on $V$. The triple $\left(v, I_{1}, G\right)$

is said to be a contextual grammar ; $\underline{V}$. is the vocabulary of the grammar, $I_{-1}$ is the base of the grammar and $G_{\text {is }}$ the con- 
textual component of the grammar.

Let us denote by $\tau_{T}$ the contextual grammar defined by (1). Consider the smallest language $I$ on $V$, fulfilling the following two conditions

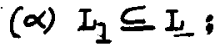

$$
\begin{aligned}
& \text { ( } \beta \text { ) if } x \in I \text { and }\langle u, v\rangle \in(G) \text {, then } u x v \in I \text {. }
\end{aligned}
$$

The language I is said to be the language generat by the contextual gramar $G$. This means that the language generated by $G$ is the intersection of all languages $I$ fulfilling the conditions $(\alpha)$ and $(\beta)$.

A language I is said to be a conteytual language if there exists a contextual gramar $G$ which generates $I$.

Proposition I. Every finite language is a contextual language.

Proof. Let $V$ be a vocabulary and let I be a finite lan. guage on $V$. It is obvious that the contextual grammar $\left(v, L_{1}, 0\right)$, where $v$ denotes the void set of contexts, generates the language $I_{I}$. The same language may be generated by means of the contextual gramar $\left(v, I_{1},(U)\right.$, where $(U)$ is formed by the num context only.

Two cont extual grammars are called equivalent if they generate the same language. The grammars $\left(V, I_{1}, 0\right)$ and $\left(V, I_{1}, U\right)$ are equivalent, since they both generate the language $I_{1}$

The converse of Proposition 1 is not true. Indeed, we have

Proposition 2. The universal language is a cont extual language.

Proof. Let $V=\left\{a_{1} a_{2}, \ldots, a_{n}\right\} \cdot$ Denote bJ $I$ the univer. sal language on $v$ : Let us put $I_{1}=\{\dot{\omega}\}$ and $\mathscr{G}=\left\{\left\langle\omega, a_{1}\right\rangle\right.$. 
$\left\langle\omega, a_{2}\right\rangle, \ldots,\left\langle\omega, a_{p}\right\rangle$. It is easy to see that the grammar $\left(V, I_{1}(G)\right.$ generates the universal language on $v$. Remarks. If we put, in the proof of Proposition $2, I_{I}=V$. instead of $I_{1}=\{\omega\}$, then the grammar $\left(V_{2} I_{2},(\mathcal{G})\right.$ does not $\theta^{\theta-}$ nerate the universal language on $V$, since the language it generates does not contain the null-string.

In order to illustrate the activity of the grammar (V,II. (C) defined in the proof of Proposition 2, let us consider the particular case when the vocabulary is formed by two elements only : $V=\{a . b\}$. The general form of a string $x$ on $V$ is

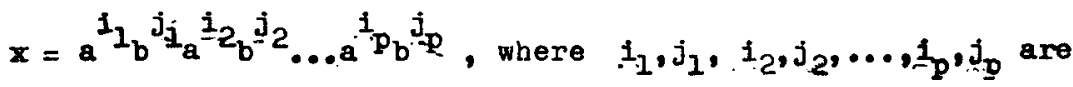
arbitrary non-negative integers. In order to generate the string x. start with the nullstring w and we apply $1_{-2}$ times the context $\langle\omega, a\rangle$. The result of this operation is the string $a^{i_{1}}$, to which we apply $j_{l}$ times the context $\langle\omega, b\rangle$ and obtain the string $a^{i l_{b} i_{1}}$. Now we apply $1_{2}$ times the context $\langle\omega, \lambda\rangle$, then $\underline{j}_{2}$ tikies the context $\langle\omega, \underline{b}\rangle$ and we continue so althernativeiy. jinen, atter $2 p-2$ steps, we have obtained the

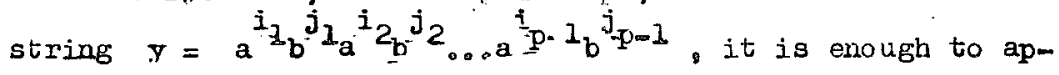
ply $i_{i}$ tices the context $\left.<, a\right\rangle$ and, to the string so obtained, $j_{p}$ times the context $\langle\omega, b\rangle$, in order to generate coupletely toe string $x$ 。

Haskell Curry consideled ine larguage $I=\left\{a b^{n}\right\}(n=1,2, \ldots)$ as a model of the set of natural numbers [5]. We call I the language of curry.

Proposition 3. The language of Curry is a contextual language. 


\section{$-\dot{4}-$}

Proof. The considered language is generated by the grammar $\left(V, I_{1},(b)\right)$, where $V=\{a \cdot b\}, I_{2}=\{a\}$ and $(\zeta)=\{\langle\omega, b\rangle\}$.

We recall that $a$ language is said to be $\hat{r}$ egular if 1 may be generated by means of a finite automaton (or, equivalentiy, by means of a finite state grammar in the sense of Ohomsky).

Proposition 4. There exists a contextual language which is not regular.

Proof. Iet us consider the language $I=\left\{a^{n} b^{n}\right\} \quad(n=1,2, \ldots)$ If we put $V=\{a, b\}, I_{1}=\{a b\}$ and $(G)=\{\langle a, b\rangle\}$, then it is easy to see that $I$ is generated by the contextual grammar $(V$, $I_{1},(C)$. Un the other band, $I$ is not a regular language. This fact was asserted by Chomsky in [3] and [4], but the proof he gives is wrong. A correct proof of this assertion and a,discussion of Chomsky's proof were given in [8], and [9].

Propositions 2,3 and 4 show that there are many infinite Ianguages which are contextual. This fact may be explained by means of

Proposition 5. If the set In is non-void and if the set (G) contains at least one non-null context, then the contextual gramnar (V. $I_{1}$, (G) generates an infinite language.

Proof. Since $L_{2}$ is non-void, we may find a string $x$ beloinging to $I_{1}$. Since (G) contains, at least one non-null context, let $\langle u, v\rangle$ be a non-null context belonging to (G) . From these assumptions, we infer. that the strings uxv. $u^{2} x v^{2}, \ldots . v^{n} \cdot x v^{n}, \ldots$ are mutually distinct and belong all to the language generated by the grammar (V,I, $(\zeta)$. Thus, this language is infinite. The converse of Proposition 5 is true. Indeed, we have 
Proposition 6. If the contextual grammar ( $V, I_{1}$ (f) gene rates an infinite language, then I is non-void, whereas $\left(C_{1}\right.$ contains a non-null context.

Proof. Let $I$ be the language generat ed by $\left(v, I_{l}(6)\right.$. If $I_{2}$ is void, $I$ is void too, hence it cannot be infinite. If (C) contains no non-null context, we have $I=I_{l}$. But $I_{1}$ is in any case finite ; thus, $I$ is finite, in contradiction with the hypothesis.

Since there are contextual language which are not regular (see Proposition 4 above), it would be interesting to establish whether all contextual languages are context-free languages. The answer is affirnative:

Pronosition 7. Every contextual language is a cont ext-free language.

Proof. Iet $L_{-}$be a contextual language. If $I$ is finite, it is a regular language. But it is well known that every regular language is a context-free language. Therefore, I is a context-free language. Nowe let us suppose that $I$ is infinite. Denote by $G=\left(V, I_{I},(G)\right.$ a contextual grammar which generates the language I. In view of Proposition 6, I 1 is non-void, whereas there exists an integer $i, 1 \leqslant 1 \leqslant p$, such that the con-

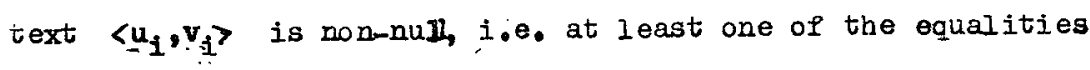
$u_{i}=\omega, v_{i}=\infty$ is false. Iet us make a choice and suppose that $u_{1} \neq \omega$ : Iet $I_{1}=\left\{x_{1}, x_{2}, \ldots, x_{n}\right\}$ and $(G)=\left\{\left\langle\underline{u}_{1}, v_{1}\right\rangle\right.$,

$\left.\left\langle u_{2}, v_{2}\right\rangle, \ldots,\left\langle u_{p}, v_{p}\right\rangle\right\}$. We define a context-free grammar $\Gamma$ as follows. The terminal vocabulary of $\Gamma$ is $v$. The non terminal vocabulary of $\Gamma$ contains one element only- denoted by s. - which is, of course, the axiom of the grammar $\Gamma$. The ter- 
minal rules of $\Gamma$ are

$$
\begin{aligned}
& s \rightarrow x_{1}: \\
& s \rightarrow x_{2} \\
& \dot{s} \rightarrow x_{m}
\end{aligned}
$$

whereas the non-terminal rules are

$$
\begin{aligned}
& s \rightarrow u_{1} s v_{1}, \\
& s \rightarrow u_{2 s} v_{2}, \\
& s \cdot \cdot \cdot \cdot \cdot \\
& s \rightarrow u_{p} s v_{p} .
\end{aligned}
$$

It is obvious that the number of terninal rules is equal to the number of strings in $I_{\mathfrak{I}}$, whereas the number of non-terminal ruies is preciseiy the number of contexts in (6). Among the nonterwinal rules, there is one at least which is non-trivial : it is the rule $s \rightarrow u_{i} S$. $v_{i}$, where $u_{j} \neq \omega$.

It is not difficult to prove that the gramnar $\Gamma$ generates the given Ianguage $T_{\text {. }}$ Indeed, the general form of a string in I is

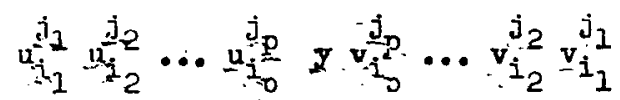

where $y \in V$ and

$$
\left\langle\underline{u}_{i_{a}}, \underline{v}_{\underline{i}_{s}}\right\rangle \in(G) \text { for } s=1,2, \ldots, \underline{p} \text {. }
$$

In order to generate the considered string we begin by aplying $\underline{j}_{1}$ times the rule

$$
s \rightarrow u_{i_{1}} \& \underline{v}_{i_{1}}
$$

In this way, we obtain the expression

$$
u_{-1}^{j_{1}} \text { S. } v_{i_{1}}^{j_{1}}
$$

The next step consists in applying ja times tbe rule 


$$
\underset{s \rightarrow u_{s_{2}} s v_{d_{2}} .}{-7-}
$$

which flelds the expression

$$
u_{j_{1}}^{j_{1}} u_{i_{3}}^{j_{2}} s v_{j_{2}}^{j_{2}} v_{i_{1}}^{j_{1}}
$$

Continuing in this way, we arrive, after p-l steps, to the expression

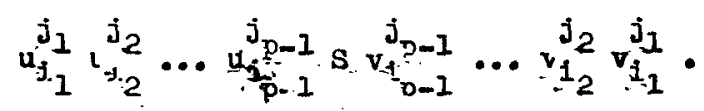

We now apply $j_{p}$ times the rule

$$
s \rightarrow u_{i p} s v_{v_{p}}
$$

and thus we obtain the expression

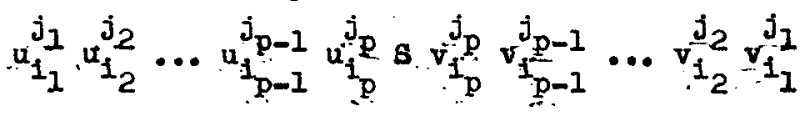

where, by applyine the terwinal rule

$$
\mathrm{S} . \longrightarrow \text { ע, }
$$

the considered string is conpletely generated. Thus, we have proved that $I$ is contained in the langugge generat ed by $\Gamma$. Conversely, let $z$ be a string generated by $\Gamma$. The ge neral form of this generation involves several consecutive applications of non-terminal rules (the number of these applications may be eventually equal to zero) followed by one and only one application of a terminal rule. It is easy to see that the result of this generation is always a string of the form (2). Thus we have proved that the language generated by $\Gamma$ is contained in $I$ : In view of the preceding considerations, I is precisely the language generated by $\Gamma$.

Proposition 7 easily permits to obtain sinple examples of 
languages which are not contextual 1anguages. For instance, the language of. Kleene $\left\{a^{n^{2}}\right\}(n=7,2, \ldots)$, the first example of an infinite langaage which is not regular, is a very aimple example of noncontextual language. It is enough to remark that the equence $\left\{n^{2}\right\}(n=1,2, \ldots)$ contains no subsequence which is an infinite arithnetic progression (We have $(n+1)^{2}-n^{2}=2 n+1$ and $\lim _{n \rightarrow \infty}(2 n+1)=\infty$, therefore for every subsequence of $\left\{\underline{n}^{2}\right\}$ the difference of two consecutive terms has the limit equal to +o when $n \rightarrow \infty$ ). But a resurt of [1] asserts, aliong others, that given an infinite context-free language $I$, the set of integers which represent the lengths of the strings in I contains an infinite arithmetic progression. It follows That the jangluage of Kleene is not context-free and, in view of Proposition 7, it is not a contextual language. The same fact follows from theorem 3.1 .2 of $[6]$, p.86.

A natural question now arrises : Do there exist non-contextual langliages awong context-free languages ? The affirmative ansmer follows froiu the following remark:

The converse of Proposition 7 is not true. Indeed, we have Proposition 8. There exists a cont ext-free language which is not a cont extua] language.

Proof. Let $v=\{a, h\}$. In view of a tbeorem of Grukke [7] there exists, for every positive integer n, a context-free language $I_{n}$ on $V$, such that every context-free grammar of $I_{n}$ contains at least $\underline{n}$ non-terminal symbols. But, as we can see in the proof of Proposition 7, every contextual language may be generated with a context-free grammar containing only one non-terminal symbol. Therefore, if $\underline{n} \geqslant 2$, $\underline{I}_{\mathfrak{n}}$ is not a contextual language. Proposition 8 guggests the natural question whetber there exist regular languages which are not cont extual languages. The 
answer is affirmative:

Pronosition 9. There exists a regular language which is not a contextual language.

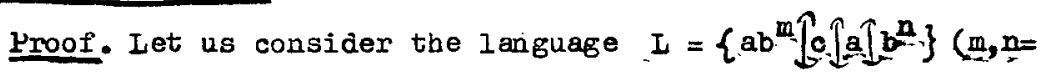
$=1,2, \ldots)$, which was used H.B.Curry $[5]$, in order to describe the set of mathelutical (true or not) propositions. This language is reguiar, since it can be generated by the rules $s \rightarrow A b, A \rightarrow A b$, $\mathrm{A} \rightarrow \mathrm{Ba}, \mathrm{B} \rightarrow \mathrm{Cc}, \mathrm{C} \rightarrow \mathrm{Cb}, \mathrm{C} \rightarrow \mathrm{Db}, \mathrm{D} \rightarrow \mathrm{a}$. We sháll show that $\mathrm{I}$ is not a contextual language. Indeed, let us admit that the contrary holds and let $\mathrm{G}=\left\langle\underline{\mathrm{V}}, \mathrm{I}_{I}, G\right\rangle$ be a contextual grammar of $\underline{\mathrm{I}}$. Here, the generai form of a string in $I$. is

$$
u_{n}^{p_{n}} \ldots \underline{u}_{2}^{p_{2}}{ }_{u_{1}}^{p_{1}} \times v_{1}^{p_{1}} \underline{v}_{2}^{p_{2}} \ldots v_{\underline{n}}^{p_{n}}
$$

where $x \in I_{1}$, whereas $\left\langle u_{i}, v_{i}\right\rangle \in()(i=1,2, \ldots, n)$ and $p_{1}, p_{2}, \ldots$ $\ldots, p_{n}$ are arbitrary positive integers. This means that $u_{1}, u_{2}, \ldots$ $\ldots, \underline{u}_{n}, v_{1}, v_{2}, \ldots, v_{n}$ in the expression (3) are formed only by those elewents of $y$ whose number of occurences in the strings of $I$ is unifiited. Unly $h$ satisfies this requirenent. It follows that in any string of I both occurrences of a and the occurence of $c$ are terms of the string $\underline{x}$ in (3). But this implies that the intermediate terms between the occurrences of a are terms of $x$, bence we can find two strings $y$ and $z$ such that

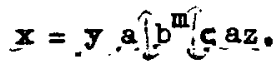

The string $y$ is obviously the nultstring $w$, whereas $z$ is of the form b? hence.

$$
x=a b^{m} \underline{m} a b .
$$

But ‥ may be here an arbitrary positive integer. Therefore, since 
$x \in I_{1}$, it follows that $I_{1}$ is an infinite set of etrings. This fact contradicts the assumption concerning $G$; is not a contextual language and Proposition 9 is proved.

The contextual grammars my be generalized. In order to generate some languages which are not context-free.

A generalized contextual grammar is a quadruple $G=\langle V$, $\left.I_{1}, I_{2}, \mathscr{C}\right)$, where $V, I_{2}$ and $\mathscr{C}$ have the same meaning as in the definition of a contextual gramar, whereas $I_{2}$ is a finite set of strings on the vocabulary $V$. We define the language $I_{G}$ generated by $G$, in the following way : I $I_{G}$ is a lamguage on $V^{-}$ and $x \in I_{y}$ if and only if we may express $x$ in the form

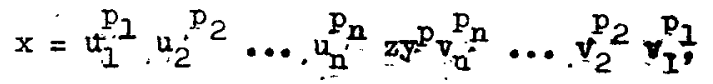
where $z \in I_{1}, y \in I_{2},\left\langle u_{i}, v_{i}\right\rangle \in G$ for $i=I, 2, \ldots, n$ and $p_{1}, p_{2}, \ldots, p_{n}, p$ are positive integers such that $p_{1}+p_{2} \ldots+p_{n}=p_{\bullet}$ Every language generated by a generalized contextual grammar is said to be a generalized contextual language.

If, in the detinition of $G$, we take $I_{2}=\{\omega\}, G$ is equivalent to a contextual grammar ; the language. $\tau_{f x}$ is then precisely the language generated by, the contextual grammar $\left\langle V, I_{1},(\mathscr{G})\right.$. Indeed, the general form of a string in the contextual language generated by $\left\langle v_{1}, I_{2},(G)\right\rangle$ is

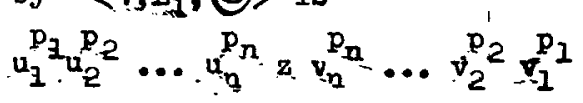

where $\left\langle\underline{u}_{1}, \underline{v}_{1}\right\rangle \in(6)(i=1,2, \ldots, n)$ and $z \in I_{1}$. We have thus proved

Proposition 10. Hery cont extual language is a generalized contextual language. 
We may consider a contextiual grammar as a particular case of generalized contextual grammar, by identifying the contextual grammar $<V, I_{1}$ ( ) with the generalized contextual grammar $<., I_{I}$, $\{\omega\}$.

It is interesting to point out that somet imes a contextual language may be easy generated by a generalized contextual grammar which is not a contextual grammar. For instance, let us consider the language $I=\left\{a^{n} b^{n}\right\}(n=1,2, \ldots)$. In view of the proof of Proposition 4, $I$ is a contextual language. We may generate I by the generalized contextual grammar (which is not a contextual grammar) $\left\langle v, I_{1}, I_{2}, G\right\rangle$, where $V=\{a, b\}, I_{1}=$ $\{\omega\}, \underline{I}_{2}=\{n\}, \zeta=\{a, \omega\}$. It is known that $\dot{t}$ is not re: gujas. We may give a similar example, with a language which is regular. In this respect let us consider the language of curry $\left\{b^{n}\right.$ \}? In view of Proposition 3, it is a contextual language. It is a regular language too, since it may be generated by the regular gramar containing the following two rules $: S \rightarrow S b$ and $S \rightarrow a_{0}$ Now let us consider the generalized contextual grammar $<v_{1} I_{1}$, $\left.I_{2}, \xi\right\rangle$, where $v=\{a, b\}, . I_{1}=\{a\}, I_{z}=\{b\} \quad G=\{\langle\omega, \omega\rangle\}$. This grammar generates the language of curry, but it is not a coniextual gramar.

Now let us show that genexalized contextual languages are an effective generalization of contextual languages.

Proposition 11 . There exista a generalized contextual language which is not a contextual language:

Proof. Let us consider the language $I=\left\{x^{n} n_{0}^{n}\right\} \quad(n=1,2, \ldots)$ It is known that this language is not context-free (see,for instance,[6],p.84). In view of proposition 7 , every contextual 
language is a context-free language; bence. I Is not a contextual language. Now let us consider the generalized conteatual grammar $G=\left\langle v, \tau_{1}, I_{2},(b)\right\rangle$, where $V_{-}=\{a, n\}, I_{2}=\{w\}, I_{0}=\{b\}$ and $G)\{\langle a ; a\rangle\}$. It is easy to see that $G$ generates the ianguage $T_{\text {. }}$

From the proof of Proposition 11 it follows immediately:

Proposition 12. Phere exists a generalized contextual language which is not a context-fice language.

We unay now ask whether the converse of Froposition 12 is true. The answer is given by

Proposition 13. There exists a context-free languge (and even a regular language) which is not a generalized contextual language.

Proof. We may consider the language $I=\left\{a b^{m} \subseteq a b^{n}-\right\}(n, n=$ $=1,2, \ldots)$ used in the proof of Proposition 9: It was showed in the proof of Proposition 9. that $I$ is regular. Let us admit that $I$ is a generalized contextual language. Given a string $x$ in I, its representation is of the form

$$
a b^{n} c a b^{p}=u_{1}^{p_{1}} u_{2}^{p_{2}} \ldots u_{n}^{p_{n}}=y^{p} v_{n}^{p_{n}} \ldots v_{2}^{p_{2}} v_{1}^{p_{1}}
$$

where $\left\langle u_{i}, v_{i}\right\rangle \in \Theta(i=1, \ldots, n), z \in I_{1}, y \in I_{2}, p_{1}+\ldots+p_{n}=p$, and $G=\left\langle V, I_{1}, I_{2}, Q\right\rangle$ is the grammar of $I_{2}$. By a reasoning sibiliar to that used in the proof of Proposition 9, we find that for. every positive integer $m$ there exists a string $z$ in $I_{1}$ such that

$$
z=a b^{m}-c a b^{8},
$$

where $s$ is a non-negative integer, depending of z. But this means that $I_{1}$ contains infinitely many strings. This fact contradicts the definition of a generalized contextual grammar. It 
follows that $I$ is not a generalized cont extual languäge.

It is to be expected that every generalized contextual language is a context-sesitive language. But the construction of the corresponding context-sensitive grammar seems to be very couplicated, if we think to the generation of the language $\left\{a^{n} b^{n} a^{n}\right\}$.

Iu.A.Šreider has introduced a new type of grammars, called neighborhood graidars (okrestnostnye grammatiki) and defined in

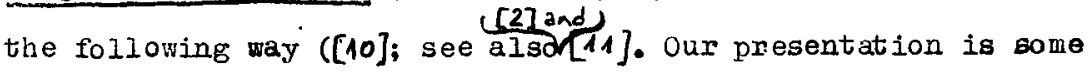
what different). Given a finite set $V$ called vocabulary, two strings $x$ and $y$ on $y$, and a context $\langle\underline{u}, v\rangle$ on $v$, we say that the pair $\{\langle u, v\rangle, y\}$ is a neighiorhood of $y$ with reapect to $x$ if we can find two strings $z$ and $w$, such that $x=24 y w$. Bvery pair of the foru $\{\langle u, v\rangle, \mathbb{Z}\}$, where $\langle u, v\rangle$ is a cont ext on $\mathrm{K}$, whereas $y$ is a string on $V$, is called a neighborhood on V. Let us consider an elenent $\theta$ which does not belong to V ; $\theta$ will be called the boundary elenent. A neighborhood grammar is a triple of the form $\langle v, \theta$,(J) $\rangle$, where $v$ is a vocabulafy, $\theta$ is the boundary elewent and $(\mathcal{J})$ is a finite set of neighborhoods on the vocabulary $v \cup\{\theta\}$. Let $I$ ke a laggage on $V$. We say that $I$ is generated by the considered neighborhood gramnar if in every string $x$ of the form $x=\theta y \theta$ (with $y \in I$ )-and only in such strings - there exists in (3), far every term $a_{i}$ of $x=g_{1} a_{2} \ldots a_{s}$, a neighlorhood of $a_{i}$ with respect to $x$. Neighborhood gramars are closely. related to the notion of context, since this notion occurs in the definition of a neighborkood. There is another notion, due to Ja.P.I.Vasilevskil and 


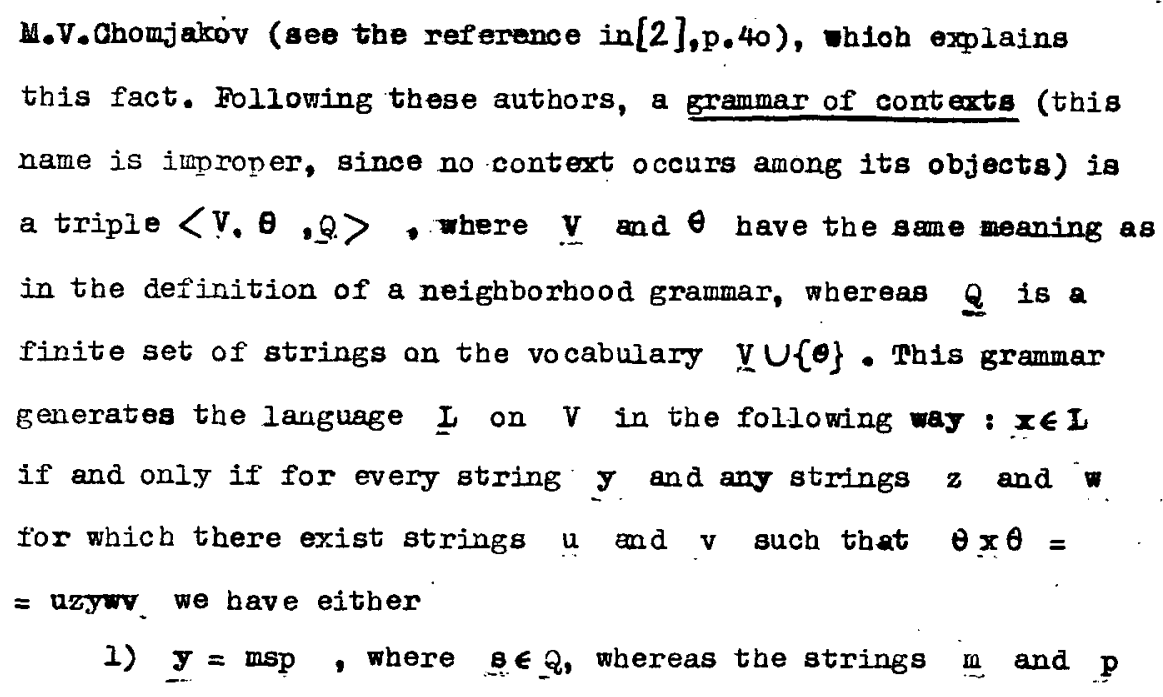
liay be eventually null

or

2) $\theta \times x=$ arynt, where $q x=2, \underline{n t}=\underline{x}$ and $r y n$ is a string belongiag to Q.

A string belousing to $Q$ is said to be closed fron the left (from the right) if its first (last) term is $\theta$. A string belonging to $Q$ is said to be closed if it is closed both from the left and fom the right.

A gramar of contexts is said to be k-bounded if every non-closed string of $Q$ is of length $\underline{k}$, whereas every closed string of $Q$ is of length not greater than $k$.

An important theorem of Borgrev asserts the equival ence between languages generated $b_{\forall}$ neighborhood grammara and languages generated by k-bounded grammars of contexts $([2], p \cdot 40)$.

Since Erammars of contexts and contextual grammars have some similarities in their definstions, it is interesting to estar blish more exactly the relation between them. 


$$
-15-
$$

Proposition 14. There exists a cont extual languase phioh is regular, but which is not a neighborhood language.

Proof. Let us consider the language $I=\left\{2^{2 n}\right\}(n=1,2, \ldots)$. This language is regular, since it is generated by the regular gramar consisting in the rules $\mathrm{S} \rightarrow \mathrm{Ta}, \mathrm{T} \rightarrow \mathrm{Ua}, \mathrm{U} \rightarrow \mathrm{Ta}$, $\mathbb{T} \rightarrow a$, where $s$ is the start symbol, $\{a\}$ is the terminal vocabulary, whereas $\{\mathrm{S}, \mathrm{T}, \mathrm{U}\}$ is the non-terninal vocabulary. Let us consider the contextual gramar $G=\langle\{a\},\{\omega\},\{\langle a, a\rangle\}\rangle$. It is exsy to see that $G$ generates the language $I$ therefore

I Is a costext ual language.

We shall show that $I$ is not a neighborhood language. In this respect, our wethod will be the following. We shall consider all systens of possible neighborhoods of the terms of the string taa a and we shall show that every such system is either a system of neighborhoods of the torals of every string $\theta a^{n} \theta \quad n=$ $=2,3,4, \ldots)$ or it is not a system of nejghborhoods of the terms of the string $\theta a^{4} \theta$. It is easy to see that the first term of the string $\theta$ aa $\theta$ adinits tbe following neighborboods : 1) $\underline{\theta}$, 2) $\underline{\theta} \underline{a}$, 3) $\underline{\theta} \underline{a} a, 4) \underline{\theta} a a \theta$. The second term has the nelghborboods : 1) $\theta \underline{a},{ }^{2)}$ z) ga , 4) $\underline{a} \underline{a} \theta$, 5) $\theta \underline{a a}$, 6) $\theta \underline{a} a \theta$. The neigbborhoods of the third term are : 1) taa , 2) .ag," 3) a, 4) $a \theta$, 5) $\theta a \underline{a} \theta, 6) \operatorname{ag} \theta$. phe last $\operatorname{term}$ has the

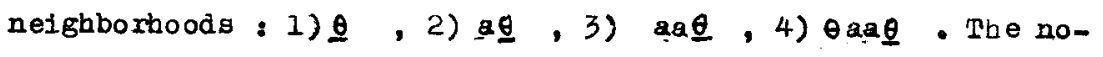
tation uxy represents hier the neighborbood $\{\langle\underline{u}, v\rangle, x\}$. It is easy to see that the fourth neighborhood of the first and of the last term cannot be a neighborbood of $\theta$ with respect to $\theta \underline{a}^{4} \theta$. On the otber hand, $\underline{a}$ is a neighborhood of $\underline{a}$ aith respect to $\theta a^{\frac{n}{2} \theta}$ for every $n=1,2, \ldots$. It follows that no 
neighborhood grammar of $I=\left\{a^{2 n}\right\}$ may contain one of the neighborhoods $\theta a^{2} \theta, \theta a^{2} \theta$ and a. Thus, if a neighborhood gram mar of I exists, it contains at least one neighborbood from every group of the following tour groups of neighborhoods :

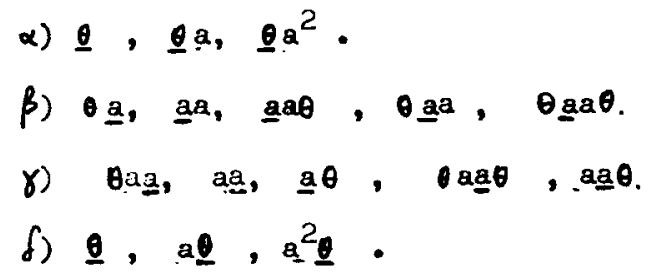

He shall consider all possible combinations between a neighborbood of the group $\beta$ and a neightorhood of the group $\gamma$. By ma ve shall deilote the combination formed by the m-th neighborhood of $\beta$ and the n-tb neighborhood of $\gamma$. It is easy to see that every neighborhood gramar containing one of the combinations $12,22,23,25,42$ generates a language which contains every string $a^{\mathfrak{n}}$ with $n \geqslant 2$. On the other hand, every neighborhood grammar contajning one of the combinations 11, 13, 14, $15,21,24,31,32,33,34,35,41,43,44,45,51,52,53,54$, 55 generates a language which either does not contain the string $a^{4}$. or contains every string $a^{n}$ vith $n \geqslant 2$. (This depends on tbe fact if the neighborhoods aa or aa belong or not to the considered neighborhood grammar). Thus, there exists no neighborhood grammar which generates the language $\left\{a \mathrm{an}^{2 n_{0}}\right.$. But the definition of (generalized) contextual grammars, though adequate to the investigation of the generative power of purely contextual operations, does not correspond to the situam tion existing in real (natural or artificial) longuages, where every string is admited only by some contexts and every context 
admits only sowe strings. Let us try to obtain a type of grammar corresponding to this more complex situation. We define a contextual grammar with choice as a system $G=\langle v, I, G, \varphi\rangle$, where V., $I_{1}$ and $(C)$ are the objects of a contextuai granmar, whereas $\varphi$ is a mapping defined on the universal language on $V$ and having the values in the set of subsets of (6). We define the language generated by $G$ as the smallest language $I$ having the following properties : $1^{\circ}$ If $x \in I_{1}, x \in I ; 2^{\circ}$ If $\quad J \in I,\langle u, y\rangle \in \varphi(y)$ and $z \in I_{1}$, then uyve $I_{1}, z \in I$ and $y z \in I$. Thus, every string chooses some contexts and every context chooses some strings. We define a contextual language with choice a language which is generated by a contextual grammar withlchoice. The investigation of these grammars and languages would better show the generative power of contextual operations, in a manner which corresponds to the situation existing in real languages. 
$\underline{\text { Referenceg }}$

1. Y.BAR-HILIEL, H.PHRL HS, B.SHAMIR : On formal properties of sirmle phrase structure grammars. Zeitschrift fur Phonetik, Sprachwissenschaft und Kommunikationsforschung, vo1.14,1961, p.143-172.

2. V.B.BORSCEV : Okrestnostnye grammatiki. Waudno-TechnjCeskaja Informacija, Serija 2, 1967, No.11, p.39-41.

3. N.CHOHSKY : Three models for the description of language.IRE Transactions on Infornation Theory, II-2, 3,1956, p.113-124.

4. iv.GHUisKY : Syntactic Structures's Gravenhage, 1957.

5. H.CURRI : Some logical aspects of grammatical structure. Prooeeãings of the Symposium in Applied iwatbematics, vol.12, Structure of language and its matheulatical aspects, Amerivith. Soc., 1961, p.56-68.

6. S.GIISBSURG : The mathenatical theory of context-free languagea. licGran-Hill Book Company, New York, 1966.

7. JGHUSKA : On a classification of cont ext-free languages. Kybernetika, vol..3, no.1, 1967, p.22-29.

8. S.MAROUS : Gramatici si automate finite. Fditura Aoademiei R.F.R., Bucuresti, 1964.

9. S.MARCUS : Sur les grammaires un nombre d' thats fini. Ca hiers de linguistique theorique et appliquee, vol.1.1965,p.147-14,

10. Ju.A.SREIDER : Okrestnostnaja model jazyka. Trud Simpoziuma po primenenijam poroźdajuscioh grammatik. Tartu, septjabr, 1967.

11. Ju.A.SREIDER : Topologiceskie medeli jazrka. Vsesojuznyi In stitut naurnoi i technixeskoi informacil, woscou, 1968. 